\title{
Cardiac tamponade following post-pericardiotomy syndrome
}

\author{
K Gandhi, ${ }^{1,2}$ iD JSK Reinders, ${ }^{1,3}$ (iD) PH Navsaria ${ }^{1}$ (iD \\ ${ }^{1}$ Trauma Centre, Department of Surgery, Groote Schuur Hospital, University of Cape Town, South Africa \\ ${ }^{2}$ Surgical Gastroenterology Unit (HPB), Department of Surgery, Faculty of Health Sciences, University of Cape Town, South Africa \\ ${ }^{3}$ Trauma Department, Groene Hart Hospital, Gouda, The Netherlands
}

Corresponding author, email: dr.karan.gandhi@gmail.com

\section{Summary}

Subxyphoid pericardial window (SPW) is performed as both a diagnostic and therapeutic intervention in patients presenting with a penetrating cardiac injury (PCI). Post-pericardiotomy syndrome (PPS) with cardiac tamponade has been reported after penetrating cardiac trauma ${ }^{1}$ and after transdiaphragmatic pericardial window. ${ }^{2}$ We describe the first PPS with acute tamponade, weeks after diagnostic SPW for a PCI.

\section{Case report}

A 25-year-old college student presented to our trauma centre after being stabbed multiple times in his head, chest and shoulder region during a robbery. He was haemodynamically stable with normal vital signs on admission. He was alert with no reported loss of consciousness. His medical history was unremarkable.

On physical examination, the patient had incised wounds to his face, scalp, right paraspinal mid-thoracic region, left parascapular region at 4 th intercostal space, left shoulder and right greater trochanteric region. The patient displayed no clinical signs of cardiac tamponade, with no distended neck veins and normal heart sounds. Chest radiograph showed no pneumo- or haemothorax and a normal left heart border. A 12-lead electrocardiogram (ECG) showed no S-T segment changes or J-waves. ${ }^{3}$ Ultrasound (US) of the heart showed a $6 \mathrm{~mm}$ pericardial effusion. Haemoglobin $(\mathrm{Hb})$ level was $13.4 \mathrm{~g} / \mathrm{dL}$.

As per institutional protocol, the patient was admitted to the trauma high-care ward for cardiac monitoring and an SPW was performed after 24 hours post-injury. At SPW, some clear pericardial fluid was evacuated, but no blood or clots were seen. The patient was discharged home the next day in a good clinical condition, only to come back a day later with complaints of fever, sweating and vomiting. On examination, his temperature was $36{ }^{\circ} \mathrm{C}$, blood pressure $129 / 66 \mathrm{mmHg}$, pulse rate $83 \mathrm{bpm}$ and respiratory rate 19 breaths per minute. All his wounds were clean. Chest radiograph, 12-lead-ECG, arterial blood gas (ABG) and blood tests revealed no abnormalities. The complaints were attributed to inadequate postoperative analgesia. He was reassured and discharged home.

Four weeks later, the patient re-presented at our trauma unit with complaints of abdominal pain, pleuritic chest pain and shortness of breath. He was apyrexial, blood pressure was $114 / 91 \mathrm{mmHg}$ with a pulse rate of $93 \mathrm{bpm}$. Respiratory rate was 16 breaths per minute. His neck veins were distended, and heart sounds were soft. His liver was enlarged, tender and palpable below the right costal margin. Chest radiograph revealed a globular heart shadow with increased cardiothoracic ratio (CTR) of 0.60 (Figure 1A). ECG displayed features of pulsus alternans (Figure 1D) and cardiac US showed a $17.6 \mathrm{~mm}$ pericardial effusion (Figure 1B) with a dilated, non-collapsible inferior vena cava (IVC) (Figure 1C). A diagnosis of post-pericardiotomy syndrome (PPS) was made, and the patient was admitted to the ward for nonsteroidal anti-inflammatory drug (NSAID) therapy. The following morning, he had persistently distended neck veins, but the chest pain felt better, so we continued NSAID treatment and close monitoring in the ward. Three days later, the patient complained of weakness and had a single episode of vomiting. He remained apyrexial and his vital signs remained stable. Repeat chest radiograph demonstrated a persistently globular heart, with a further increased CTR of 0.62 . A few hours later, the patient was found cold and unresponsive with agonal breathing in the ward. He was found to be pulseless and CPR was commenced immediately, while awaiting equipment to perform a resuscitative thoracotomy. Deep endobronchial intubation was performed, so as to enter the right main bronchus and collapse the left lung, and a left anterolateral thoracotomy was performed at the bedside. Furthermore, no medication was required to perform the intubation or the thoracotomy. The bulging pericardial sac was noted, and this was opened using the same blade that we performed the thoracotomy with. A large gush of fluid estimated between 400-500 ml was drained immediately, and open cardiac massage was commenced, with almost immediate return of spontaneous cardiac activity and circulation. By this time, the patient had had three doses of intravenous adrenaline.

Temperature measured at this point was $33.6^{\circ} \mathrm{C}$, ABG revealed severe metabolic acidosis with $\mathrm{pH}$ 6.633, and lactate $27 \mathrm{mmol} / \mathrm{L}$. The patient was rushed to the operating theatre, where antibiotics were administered and copious warm lavage of the left thoracic and pericardial cavities was 

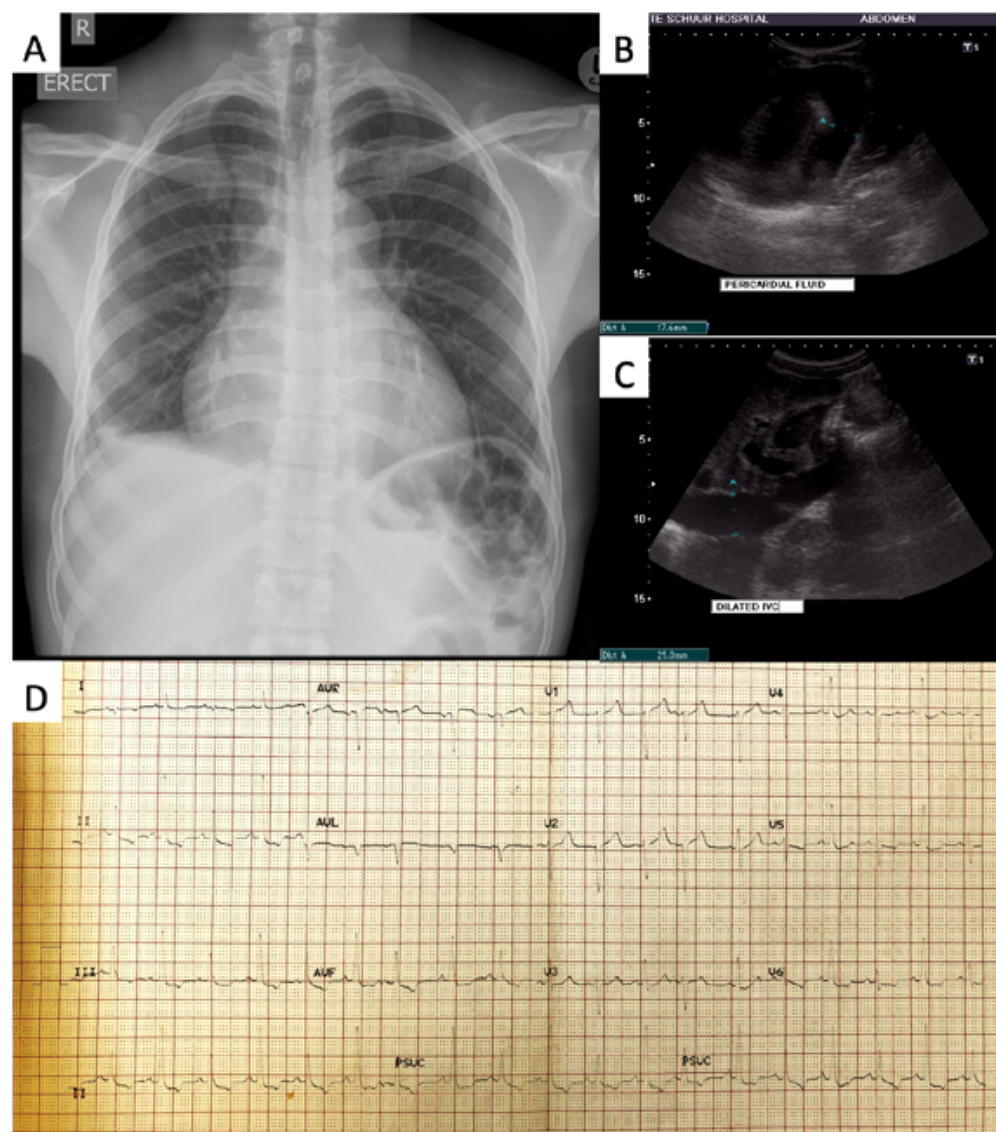

Figure 1: Radiologic and ECG findings at the time of diagnosis of post-pericardiotomy syndrome. A: Chest radiograph demonstrating a globular heart, straightened left heart border and increased cardiothoracic ratio. B: Cardiac ultrasound image demonstrating a $17.6 \mathrm{~mm}$ pericardial effusion. C: Ultrasound image demonstrating a dilated inferior vena cava. D: ECG demonstrating pulsus alternans on lead II.

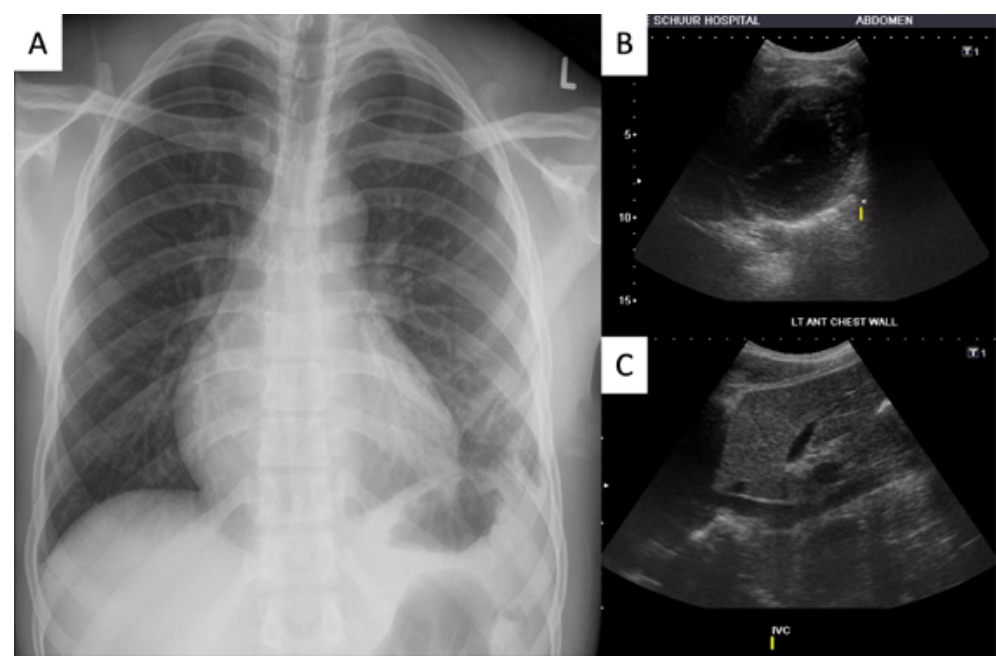

Figure 2: Follow up radiologic images. A: Chest radiograph demonstrating a normal cardiac shadow with post-thoracotomy changes. B: Cardiac ultrasound image with no demonstratable pericardial effusion. C: Ultrasound image demonstrating a non-dilated inferior vena cava.

performed, with adequate drainage and partial closure of the pericardiotomy. The thoracotomy wound was closed, and the patient was ventilated with supportive care in the intensive care unit (ICU). Inotropic support was weaned overnight, and the patient was successfully extubated the next morning, fully awake with no neurologic deficit. The chest drain was removed on postoperative day three and the patient was eventually discharged home on day five, well and pain-free after having made a complete recovery. Follow-up chest radiograph two weeks later was unremarkable (Figure 2A) and outpatient cardiac US after a month demonstrated no pericardial effusion (Figure 2B) and normal IVC (Figure 2C). He returned to college to continue his studies and is still alive and well.

\section{Discussion}

Groote Schuur Hospital, Cape Town, is a highvolume trauma centre, that sees a large number of patients presenting with stab injuries. PCI in haemodynamically stable patients is suspected when patients present with stab wounds in the cardiac box, usually associated with haemo- or pneumothorax. The protocol in this scenario entails further clinical evaluation, 12-lead ECG and radiologic investigation in the form plain chest radiography and cardiac ultrasound once the primary survey has been completed. Beck's triad (hypotension, distended neck veins and muffled heart sounds) may not be evident in haemodynamically stable patients, but certain signs like J-waves on $\mathrm{ECG}^{3}$ and pericardial fluid on cardiac US are suggestive of PCI. Patients with positive findings undergo SPW at 24-48 hours, which is the gold standard of diagnosing haemopericardium and can also be therapeutic. When positive, pericardial lavage is performed with warm saline followed by observation for any active bleeding which would prompt sternotomy. If no active bleeding is seen, pericardial drainage is performed to monitor for delayed bleeding. If SPW is negative for haemopericardium, the pericardium is left open and the fascia and skin are closed without drainage of the pericardium and patients are routinely discharged the next day. Previous data has shown that delayed SPW (within 48 hours) in stable patients is safe and may serve as adequate management in most cases, thereby avoiding sternotomy. ${ }^{4,5}$ Our patient underwent treatment as per the above protocol, at his index admission. In his case, SPW was negative and he was discharged the next day. He re-presented the following day with clinical features suggestive of PPS and again four weeks later with severe pericarditis with poor response to NSAID therapy that eventually resulted in cardiac tamponade and arrest. Despite the profound metabolic acidosis at the time of arrest, his full recovery may be attributed to the severe hypothermia at the time of resuscitation.

PPS, an inflammatory syndrome of the pericardium thought to be due to a heightened immune response to pericardial injury, has been described following various forms of cardiac trauma and surgical pericardiotomy. ${ }^{6}$ Perioperative colchicine prophylaxis has been shown in one randomised trial to reduce the incidence of PPS $^{7}$ but once established, treatment is empiric with NSAID or corticosteroid therapy. PPS may range from simple pericarditis, to pericardial effusion, atrial fibrillation and cardiac tamponade at the far end of the spectrum. A high index of suspicion should be maintained 
in patients undergoing any form of pericardiotomy, especially those who re-present with early symptoms after a procedure that is generally well-tolerated. Guidelines on the management of pericardial effusion associated with PPS are unclear in terms of the necessity, indications and timing of drainage or surgery. There is sparse data and expert consensus only recommends drainage in cases of cardiac tamponade or those refractory to medical management. Predicting progression to tamponade versus response to medical treatment remains challenging.

Miller et al. ${ }^{8}$ reported their experience with diagnostic pericardial window in 1987 and reported two cases of PPS without tamponade and successful NSAID therapy. As per our knowledge, we herewith describe the first report of a patient presenting with PPS and acute tamponade following diagnostic SPW, which has otherwise been shown to be a safe and effective procedure with low complication rates.

\section{Conflict of interest}

The authors declare no conflict of interest.

\section{Funding source}

None.

\section{ORCID}

K Gandhi (iD) https://orcid.org/0000-0003-3543-1428

JSK Reinders (iD https://orcid.org/0000-0001-5509-388X

PH Navsaria (iD https://orcid.org/0000-0002-5152-3317

\section{REFERENCES}

1. Donahoe L, Ouzounian M, MacDonald S, Field S. Delayed pericardial effusion and cardiac tamponade following penetrating chest trauma. CJEM. 2013;15(3):186-9. Available from: https://www.ncbi.nlm.nih.gov/pubmed/23663469.
2. King DR, Vlahakes GJ, Johri AM, Sheikh AY. Postpericardiotomy syndrome from transdiaphragmatic pericardial window following trauma: first description and review of the literature. J Cardiovasc Med. 2009;10(10):8069. Available from: https://www.ncbi.nlm.nih.gov/ pubmed/19606058.

3. Nicol AJ, Navsaria PH. The J-wave: a new electrocardiographic sign of an occult cardiac injury. Injury. 2014;45(1):112-5. Available from: https://www.ncbi.nlm.nih. gov/pubmed/23856630.

4. Nicol AJ, Navsaria PH, Hommes M, et al. Sternotomy or drainage for a haemopericardium after penetrating trauma: a randomised controlled trial. Ann Surg. 2014;259(3):43842. Available from: https://www.ncbi.nlm.nih.gov/ pubmed/23604058.

5. Navsaria PH, Nicol AJ. Haemopericardium in stable patients after penetrating injury: is subxiphoid pericardial window and drainage enough? A prospective study. Injury. 2005;36(6):745-50. Available from: https://www.ncbi.nlm. nih.gov/pubmed/15910827.

6. Imazio M. The post-pericardiotomy syndrome. Curr Opin Pulm Med. 2012;18(4):366-74. Available from: https://www. ncbi.nlm.nih.gov/pubmed/22487945.

7. Imazio M, Brucato A, Ferrazzi $\mathrm{P}$, et al. Colchicine for prevention of postpericardiotomy syndrome and postoperative atrial fibrillation: the COPPS-2 randomised clinical trial. JAMA. 2014;312(10):1016-23. Available from: https://www. ncbi.nlm.nih.gov/pubmed/25172965.

8. Miller FB, Bond SJ, Shumate CR, Polk HC Jr, Richardson JD. Diagnostic pericardial window. A safe alternative to exploratory thoracotomy for suspected heart injuries. Arch Surg. 1987;122(5):605-9. Available from: https://www.ncbi. nlm.nih.gov/pubmed/3579570. 